CORRECTION

https://doi.org/10.1038/s41586-019-0938-4

\section{Author Correction: Predictable and precise template-free CRISPR editing of pathogenic variants}

Max W. Shen, Mandana Arbab, Jonathan Y. Hsu, Daniel Worstell, Sannie J. Culbertson, Olga Krabbe, Christopher A. Cassa, David R. Liu, David K. Gifford \& Richard I. Sherwood

Correction to: Nature https://doi.org/10.1038/s41586-018-0686-x, published online 07 November 2018.

In this Article, a data processing error had a minor effect on Fig. 3e and Extended Data Table 2. The error was caused by a single line of code used to analyse some of the high-throughput DNA sequencing (HTS) data, which failed to re-initialize a temporary memory buffer holding data processing results for each read. This buffer was flushed at each $1 \%$ of progress during data processing on sequencing read files. This bug resulted in copying each sequence alignment a uniformly random number of times between 1 and 100 before calculating the average frequencies of each Cas9-induced mutant genotype. This erroneous code was only used in some data analysis, and we have re-analysed all such datasets with the corrected code. Owing to our experimental design choices of maintaining, on average, $>2,000$ cells per target site, and performing deep sequencing with sufficiently large depth, each unique editing outcome was independently observed many times. As a result, and by the central limit theorem, the total counts of each Cas9induced mutant genotype clustered tightly around the ratio 50.5:1, in which 50.5 is the mean value of the integer range 1-100. All downstream calculations converted counts to relative frequency (count of each unique event, divided by the total count of all events), eliminating the $50.5 \times$ overcounting. As a result, the mean frequency of each Cas9induced mutant genotype resulting from the erroneous code and corrected code were expected to be consistent given sufficiently large $n$ for the central limit theorem. For most datasets analysed with the erroneous code, the median correlation between values reported in the paper and values re-analysed with the corrected code is $>0.99$, indicating that the error had a negligible effect on the reported data. However, for the experiment that corresponds to Fig. 3e and Extended Data Table 2, in which we tested ten guide RNAs (gRNAs) predicted to induce
Corrected Fig. 3e

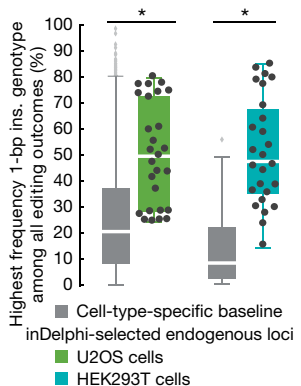

Fig. 1 This is the corrected Fig. 3e and the incorrect Fig. 3e published in the original Article.

precision-40 1-base-pair (1-bp) insertion repair profiles in the endogenous genome of human HEK293 and U2OS cells, the corrected results differ slightly from those originally reported. Figure $3 \mathrm{e}$ and Extended Data Table 2 of the original Article have been corrected, and Fig. 1 of this Amendment shows the original panels, for transparency.

As a result of the re-analysis, the sentence: "We observed that 10 out of 14 predicted precision-40 1-bp insertion gRNAs induced a single 1 -bp insertion genotype in $\geq 40 \%$ of edited products with an overall significantly higher precision $\left(P<4.2 \times 10^{-8}\right)$ than baseline data in HEK293T (median 55\% compared with 25\% baseline in VO target sites in HEK293) and U2OS cells (median 57\% compared with 14\% baseline in lib-A, U2OS, Fig. 3e)." should read "We observed that 9 out of 14 predicted precision-40 1-bp insertion gRNAs induced a single 1-bp insertion genotype in $\geq 40 \%$ of edited products with an overall significantly higher precision $\left(P<\mathbf{8 . 0} \times \mathbf{1 0}^{-4}\right)$ than baseline data in HEK293T (median $\mathbf{4 8} \%$ compared with $\mathbf{1 4 \%}$ baseline in VO target sites in HEK293) and U2OS cells (median $\mathbf{5 0 \%}$ compared with 25\% baseline in lib-A, U2OS, Fig. 3e)." (with changes highlighted in bold).

In addition, in the sentence starting "Building on this idea of precision gRNAs,..." the reported median value among edited products should be ' $49 \%$ ' rather than '61\%'. We apologize for these errors, and the altered analysis does not change any of the conclusions of the manuscript. The original Article has been corrected online. 
Corrected Extended Data Table 2

\begin{tabular}{|c|c|c|c|c|}
\hline \multirow[b]{2}{*}{$\begin{array}{l}\text { Gene, exon/chr, } \\
\text { cutsite (hg19) }\end{array}$} & \multicolumn{4}{|c|}{$\begin{array}{l}\text { Observed frequency among all edited products } \\
\text { from deep sequencing at endogenous loci (\%) }\end{array}$} \\
\hline & $\begin{array}{l}\text { Frameshift, } \\
\text { U2OS }\end{array}$ & $\begin{array}{l}\text { Most frequent } \\
\text { genotype, } \\
\text { U2OS }\end{array}$ & $\begin{array}{l}\text { Frameshift, } \\
\text { HEK293T }\end{array}$ & $\begin{array}{l}\text { Most frequent } \\
\text { genotype, } \\
\text { HEK293T }\end{array}$ \\
\hline $\begin{array}{l}\text { VEGFA } \\
\text { exon1: } 458\end{array}$ & 91,87 & $36,34^{\star}$ & 90,90 & $43,40^{\star}$ \\
\hline $\begin{array}{l}\text { VEGFR2 } \\
\text { exon5: } 2\end{array}$ & 91,91 & $50,53^{\star}$ & 91,91 & $50,24^{\star}$ \\
\hline $\begin{array}{l}\text { PDCD1 } \\
\text { exon5: } 208\end{array}$ & 90,90 & $20,21^{\star}$ & 91,90 & $29,13^{*}$ \\
\hline $\begin{array}{l}\text { APOB } \\
\text { exon25: } 147\end{array}$ & 83,83 & $22,21^{*}$ & 87,85 & $35,18^{*}$ \\
\hline $\begin{array}{l}\text { VEGFA } \\
\text { exon3: } 127\end{array}$ & 85,89 & $27,29^{\star}$ & 93,91 & $55,32^{*}$ \\
\hline $\begin{array}{l}\text { CCR5 } \\
\text { exon1: } 1941\end{array}$ & 82,81 & $20,21^{*}$ & 86,84 & $43,27^{\star}$ \\
\hline $\begin{array}{l}\text { CD274 } \\
\text { exon2: } 271\end{array}$ & 85,86 & $9,10^{\star}$ & 84,82 & $31,14^{\star}$ \\
\hline $\begin{array}{l}\text { APOB } \\
\text { exon26: } 5590\end{array}$ & 91,89 & $28,25^{\star}$ & 88 & $37^{\star}$ \\
\hline $\begin{array}{l}\text { VEGFR2 } \\
\text { exon26: } 19\end{array}$ & 82,82 & $35,33^{*}$ & 82,82 & $40,24^{\star}$ \\
\hline $\begin{array}{l}\text { CXCR4 } \\
\text { exon1: } 825\end{array}$ & 86,86 & $32,33^{\star}$ & 91 & $54^{\star}$ \\
\hline $\begin{array}{l}\overline{\text { PCSK }} \overline{-}- \\
\text { exon11: } 15\end{array}$ & 81,78 & $28,25^{\dagger}$ & 78 & $27^{\dagger}$ \\
\hline $\begin{array}{l}\text { CCR5 } \\
\text { exon1: } 885\end{array}$ & 84,85 & $55,52^{\dagger}$ & 67 & $46^{\dagger}$ \\
\hline $\begin{array}{l}\text { CCR5 } \\
\text { exon1: } 1027\end{array}$ & 92,94 & $61,60^{\dagger}$ & 91,92 & $49,58^{\dagger}$ \\
\hline $\begin{array}{l}\text { APOB } \\
\text { exon26: } 5573\end{array}$ & 93,93 & $75,74^{\dagger}$ & 93,95 & $69,81^{\dagger}$ \\
\hline $\begin{array}{l}\text { CCR5 } \\
\text { exon1: } 61\end{array}$ & 94,94 & $37,25^{\dagger}$ & 83,89 & $29,38^{\dagger}$ \\
\hline $\begin{array}{l}\text { CCR5 } \\
\text { exon1: } 1577\end{array}$ & 81,81 & $28,29^{\dagger}$ & 80,83 & $29,43^{\dagger}$ \\
\hline $\begin{array}{l}\text { APOB } \\
\text { exon22: } 100\end{array}$ & 89,89 & $25,27^{\dagger}$ & 91,89 & $23,38^{\dagger}$ \\
\hline $\begin{array}{l}\text { APOBEC3B } \\
\text { exon3: } 202 \underline{-}\end{array}$ & $\begin{array}{l}83,84 \\
---\ldots\end{array}$ & $\begin{array}{l}50,52^{\dagger} \\
-\ldots-\ldots-\ldots\end{array}$ & $\begin{array}{l}75,88 \\
--\ldots\end{array}$ & $\begin{array}{l}51,60^{\dagger} \\
---\ldots--\end{array}$ \\
\hline $\begin{array}{l}\text { MACCHC } \\
\text { chr1: } 45973892\end{array}$ & 97,95 & $80,77^{\ddagger \ddagger}$ & 97,98 & $78,85^{\dagger \ddagger}$ \\
\hline $\begin{array}{l}\text { PROK2 } \\
\text { chr3: } 71821967\end{array}$ & 93,94 & $44,41^{\text {t末 }}$ & 93,93 & $45,53^{\dagger \ddagger}$ \\
\hline $\begin{array}{l}\text { IDS } \\
\text { chrX: } 148564700\end{array}$ & 95,95 & $72,74^{\dagger \ddagger}$ & 93,95 & $64,80^{\dagger \ddagger}$ \\
\hline $\begin{array}{l}\text { ECM1 } \\
\text { chr1: } 150484936\end{array}$ & 87,89 & $44,47^{\dagger \ddagger}$ & 89,89 & $32,35^{\dagger \neq}$ \\
\hline $\begin{array}{l}\text { KCNH2 } \\
\text { chr7: } 150644566\end{array}$ & 40 & $25^{\dagger \neq}$ & 65,95 & $35,14^{\dagger \ddagger}$ \\
\hline $\begin{array}{l}\text { LDLR } \\
\text { chr19: } 11222303\end{array}$ & 90,91 & $78,77^{\dagger \ddagger}$ & 90,96 & $77,83^{\dagger \ddagger}$ \\
\hline
\end{tabular}

Original Extended Data Table 2

\begin{tabular}{|c|c|c|c|c|}
\hline \multirow[b]{2}{*}{$\begin{array}{l}\text { Gene, exon/chr, } \\
\text { cutsite (hg19) }\end{array}$} & \multicolumn{4}{|c|}{$\begin{array}{l}\text { Observed frequency among all edited products } \\
\text { from deep sequencing at endogenous loci (\%) }\end{array}$} \\
\hline & $\begin{array}{l}\text { Frameshift, } \\
\text { U2OS }\end{array}$ & $\begin{array}{l}\text { Most frequent } \\
\text { genotype, } \\
\text { U2OS }\end{array}$ & $\begin{array}{l}\text { Frameshift, } \\
\text { HEK293T }\end{array}$ & $\begin{array}{l}\text { Most frequent } \\
\text { genotype, } \\
\text { HEK293T }\end{array}$ \\
\hline $\begin{array}{l}\text { VEGFA } \\
\text { exon1: } 458\end{array}$ & 72,72 & $9,11^{*}$ & 81,71 & $28,9^{\star}$ \\
\hline $\begin{array}{l}\text { VEGFR2 } \\
\text { exon5: } 2\end{array}$ & 91,91 & $49,52^{\star}$ & 91,91 & $49,23^{\star}$ \\
\hline $\begin{array}{l}\text { PDCD1 } \\
\text { exon5: } 208\end{array}$ & 90,90 & $20,22^{\star}$ & 91,91 & $29,13^{*}$ \\
\hline $\begin{array}{l}\text { APOB } \\
\text { exon25: } 147\end{array}$ & 83,83 & $22,21^{\star}$ & 87,85 & $36,17^{\star}$ \\
\hline $\begin{array}{l}\text { VEGFA } \\
\text { exon3: } 127\end{array}$ & 86,89 & $28,30^{\star}$ & 92,91 & $56,32^{*}$ \\
\hline $\begin{array}{l}\text { CCR5 } \\
\text { exon1: } 1941\end{array}$ & 83,81 & $20,21^{\star}$ & 86,84 & $43,27^{\star}$ \\
\hline $\begin{array}{l}\text { CD274 } \\
\text { exon2: } 271\end{array}$ & 85,86 & $9,10^{*}$ & 84,82 & $31,14^{*}$ \\
\hline $\begin{array}{l}\text { APOB } \\
\text { exon26: } 5590\end{array}$ & 91,89 & $30,27^{\star}$ & 89 & $40^{*}$ \\
\hline $\begin{array}{l}\text { VEGFR2 } \\
\text { exon26: } 19\end{array}$ & 82,82 & $35,33^{\star}$ & 83,82 & $41,23^{\star}$ \\
\hline $\begin{array}{l}\text { CXCR4 } \\
\text { exon1: } 825\end{array}$ & 86,86 & $32,33^{\star}$ & 91 & $55^{\star}$ \\
\hline $\begin{array}{l}\overline{\text { PCSK}} \overline{-}- \\
\text { exon11: } 15\end{array}$ & $\begin{array}{l}---1 \\
91,89\end{array}$ & $64,64^{\dagger}$ & $\begin{array}{ll}---7+n \\
89\end{array}$ & $60^{+}$ \\
\hline $\begin{array}{l}\text { CCR5 } \\
\text { exon1: } 885\end{array}$ & 90,91 & $74,71^{\dagger}$ & 78 & $65^{\dagger}$ \\
\hline $\begin{array}{l}\text { CCR5 } \\
\text { exon1: } 1027\end{array}$ & 92,94 & $62,62^{\dagger}$ & 91,92 & $50,60^{\dagger}$ \\
\hline $\begin{array}{l}\text { APOB } \\
\text { exon26: } 5573\end{array}$ & 93,93 & $75,74^{\dagger}$ & 93,95 & $69,82^{\dagger}$ \\
\hline $\begin{array}{l}\text { CCR5 } \\
\text { exon1: } 61\end{array}$ & 94,92 & $21,16^{\dagger}$ & 84,88 & $19,28^{\dagger}$ \\
\hline $\begin{array}{l}\text { CCR5 } \\
\text { exon1: } 1577\end{array}$ & 81,81 & $29,30^{\dagger}$ & 80,84 & $29,46^{\dagger}$ \\
\hline $\begin{array}{l}\text { APOB } \\
\text { exon22: } 100\end{array}$ & 89,90 & $28,31^{\dagger}$ & 90,89 & $26,40^{\dagger}$ \\
\hline $\begin{array}{l}\text { APOBEC3B } \\
\text { exon3: } 202 \\
\end{array}$ & 83,83 & $52,54^{\dagger}$ & 74,87 & $52,62^{\dagger}$ \\
\hline $\begin{array}{l}\overline{M A C} \bar{C} \overline{H C} \\
\text { chr1: } 45973892\end{array}$ & $\begin{array}{l}--- \\
97,95\end{array}$ & $81,77^{\dagger \ddagger}$ & $\begin{array}{l}---- \\
97,98\end{array}$ & $\begin{array}{l}79,86^{\dagger \ddagger} \\
\end{array}$ \\
\hline $\begin{array}{l}\text { PROK2 } \\
\text { chr3: } 71821967\end{array}$ & 92,93 & $45,45^{\dagger \neq}$ & 92,93 & $49,58^{\dagger \ddagger}$ \\
\hline $\begin{array}{l}\text { IDS } \\
\text { chrX: } 148564700\end{array}$ & 96,95 & $73,76^{\dagger \ddagger}$ & 93,95 & $63,79^{\dagger \ddagger}$ \\
\hline $\begin{array}{l}\text { ECM1 } \\
\text { chr1: } 150484936\end{array}$ & 87,89 & $47,52^{\dagger \ddagger}$ & 88,89 & $33,37^{\dagger \ddagger}$ \\
\hline $\begin{array}{l}\text { KCNH2 } \\
\text { chr7: } 150644566\end{array}$ & 46 & $30^{\dagger \neq}$ & 89,93 & $71,75^{\dagger \ddagger}$ \\
\hline $\begin{array}{l}\text { LDLR } \\
\text { chr19: } 11222303\end{array}$ & 91,92 & $79,78^{\dagger \ddagger}$ & 90,96 & $78,84^{\dagger \ddagger}$ \\
\hline
\end{tabular}

Fig. 2 This is the corrected Extended Data Table 2 and the incorrect Extended Data Table 2 published in the original Article. 